\title{
EDITORIAL
}

\section{Providing mental health services in poverty}

\author{
David Skuse $\odot$
}

Professor of Behavioural and Brain Sciences, Division of Population, Policy and Practice, UCL Great Ormond Street Institute of Child Health, London, UK. Email d.skuse@ucl.ac.uk

Keywords. Mental health; poverty; community care; inequality;

mhGAP

First received 3 Nov 2021 Accepted 3 Nov 2021

\section{doi:10.1192/bji.2021.56}

(c) The Author(s), 2022. Published by Cambridge University Press on behalf of the Royal College of Psychiatrists. This is an Open Access article, distributed under the terms of the Creative Commons Attribution licence (https:/lreativecommons. (https.//creativecommons.org/ licenses/by/4.0/), which permit unrestricted re-use, distribution, and reproduction in any medium provided the original work is

properly cited.
All too often, we reflect in this journal on the impoverishment of psychiatric care around the world, relative to the staffing of general medical services. The relatively limited funds that are made available for mental health, as a proportion of the national budget of most countries, never reflects the magnitude of the need, nor the potential cost to national productivity of having so many untreated or undertreated individuals. But some mental health services are in poverty because the country as a whole is in a state of impoverishment.

Jude Mary Cénat and colleagues report on the situation in Haiti, where one in four of the population survives on less than $\$ 1.25$ per day. ${ }^{1}$ They focus on the need to provide mental healthcare to the country's North Department, and on the help provided by the World Health Organization's (WHO's) mhGAP programme. Valuable advice is given for policy makers on how to provide community-based, cost-effective care.

Nepal is an equivalently poor country in which one in six of the population lives on less than $\$ 1.9$ per day, and mental healthcare receives less than $1 \%$ of the healthcare budget. Rakesh Singh and colleagues review the history of psychiatric services in Nepal and discuss the recent decision to develop a National Mental Health Strategy. ${ }^{2}$ Interestingly, a plan to roll out community mental health services was inaugurated in 1983, and these have now been extended throughout the country with the support of non-government organisations (NGOs).
Paraguay is, in contrast, a middle-income country, but $6 \%$ of the population lives on less than $\$ 3.2$ a day. The uneven distribution of mental health services in that country is described by Anne Aboaja and colleagues. ${ }^{3}$ They comment that, despite an intention to increase communitybased care dating back to 1990, most targeted mental health funds still go primarily to hospital services. As in so many other countries with pockets of poverty in their rural populations, considerable mental health inequalities persist.

I have here outlined a range of different approaches to dealing with psychiatric care in conditions of absolute or relative poverty: there is plenty of scope for our readers to learn from one another about the best way of tackling such inequalities at minimal cost.

\section{Declaration of interest}

D.S. is Editor of BJPsych International.

\section{References}

1 Cénat J, Harerimana B, Michel G, McIntee S, Mukunzi J, Hajizadeh S, et al (2021) The global challenge of providing mental health services in poverty: the situation of Northern Haiti. BJPsych Int; doi:10.1192/bji.2021.22

2 Singh R, Gupta A, Singh B, Basnet P, Arafat S. (2021) History of psychiatry in Nepal. BJPsych Int; doi:10.1192/bji.2021.51

3 Aboaja A, Wahab A, Cao Y, O'Higgins M, Torales J. (2021) Mental health in the republic of Paraguay. BJPsych Int; doi:10.1192/bji.2021.24 